\title{
TERAPIA ASSISTIDA POR ANIMAIS: O ANIMAL COMO AUXÍLIO TERAPÊUTICO
}

\author{
Gabriela Pena de Oliveira ${ }^{1}$ \\ Silvia Helena Modenesi Pucci ${ }^{2}$
}

RESUMO: Há muitas medidas de promoção da saúde e a Terapia Assistida por Animais (TAA) é classificada como umas destas medidas. Desta forma, este trabalho aborda a perspectiva da utilização do animal como auxílio terapêutico através da TAA. O objetivo principal foi verificar como a Terapia Assistida por Animais auxilia o desenvolvimento das habilidades sociais na infância $e$ adolescência. Como metodologia, foi realizada uma pesquisa bibliográfica com a utilização das bases de dados indexadas. Os resultados mostraram que a TAA apresenta-se como uma intervenção que facilita o processo de socialização das crianças e adolescentes, facilitando o estabelecimento da comunicação e a melhora das relações interpessoais. Portanto, a TAA se apresenta como uma intervenção terapêutica potencial, entretanto, mediante a limitação de trabalhos sobre o tema, se faz necessário mais estudos que evidenciem a relação da TAA com as habilidades sociais.

Palavras-Chave: TAA. Habilidades sociais. Infância. Adolescência.

ABSTRACT: There are many health promotion measures and Animal Assisted Therapy (AAT) is classified as one of these measures. Thus, this work addresses the perspective of using the animal as a therapeutic aid through AAT. The main objective was to verify how Animal Assisted Therapy helps the development of social skills in childhood and adolescence. As a methodology, a bibliographic research was carried out using indexed databases. The results showed that AAT presents itself as an intervention that facilitates the socialization process of children and adolescents, facilitating the establishment of communication and the improvement of interpersonal relationships. Therefore, AAT presents itself as a potential therapeutic intervention, however, due to the limitation of studies on the subject, further studies are needed to show the relationship of AAT with social skills.

Keywords: AAT social skills. Childhood. Adolescence.

\footnotetext{
${ }^{\mathrm{I}}$ Graduanda em Psicologia. UNISA - Universidade Santo Amaro.

${ }^{2}$ Prof Dr. UNISA - Universidade Santo Amaro
} 


\section{INTRODUÇÃO}

Atualmente há muitas medidas de intervenção para promoção da saúde e a Terapia Assistidas por Animais (TAA) é considerada como uma destas medidas (MARINHO E ZAMO, 2017).

A TAA é um modelo de terapia que tem o animal como base no processo terapêutico, esta intervenção tem sido aplicada para estimular o desenvolvimento de pessoas com problemas emocionais, sociais, cognitivos e físicos (CAPOTE E COSTA, 20II). Dotti (2005), ressalta que a TAA apresenta contribuições voltadas a melhora da fala, cognição, autoestima, autocuidados, socialização e melhora do desenvolvimento físico.

As habilidades sociais se referem a expressão de opiniões, atitudes, sentimentos e desejos, habilidades estas que facilitam o estabelecimento das relações interpessoais (CABALLO, 1996). De acordo com Del Prete e Del Prete (2009), alguns comportamentos como a empatia, assertividade, cooperação, autocontrole e a comunicação, facilitam o aperfeiçoamento social da pessoa.

Além do bem-estar da pessoa através da interação com o animal, é necessário atenção ao bem-estar do animal nas sessões de TAA, logo, a relação paciente e animal deve ser considerada a partir de uma perspectiva holística, considerando o bem-estar e as necessidades de todos os envolvidos no processo (ROCHA, 2015).

Diante disso, o presente trabalho teve como objetivo verificar como a TAA auxilia o desenvolvimento das habilidades sociais na infância e adolescência. Desta forma, mediante os resultados apresentados, faz-se necessário a continuidade de pesquisas sobre a relação da TAA com o desenvolvimento das habilidades sociais, visto que foram localizados poucos trabalhos sobre o tema, porém, mesmo com a limitação de trabalhos, foi possível perceber que esta intervenção apresenta benefícios no que tange as habilidades de comunicação e desenvolvimento das relações interpessoais, demonstrando-se como uma intervenção de tratamento potencial. 


\section{TERAPIA ASSISTIDA POR ANIMAIS}

De acordo com Ferreira e Gomes (2017), a Terapia Assistida por Animais (TAA) é um modelo de terapia utilizada na psicologia que tem o animal como base no processo terapêutico; os objetivos da técnica são pré-definidos, como por exemplo, considerar o perfil do paciente, demonstrar ideia clara sobre a aplicabilidade dos animais no tratamento e proporcionar saúde emocional, física e social. Ainda de acordo com os autores, a intervenção por meio dos animais pode dar-se através de diferentes configurações, sendo individual ou em grupo, aplicando-se em diversas faixas etárias e em vários contextos.

Entre os critérios da aplicação da técnica, deve-se considerar os objetivos da mesma, sendo necessário a elaboração de relatórios a cada atendimento, além de seguir um protocolo estipulado, no qual dentro deste protocolo inclui-se o acompanhamento e avaliação do animal por um médico veterinário, para que então o animal seja liberado a realizar as visitas (PEREIRA et al., 2007).

Outro ponto a ser verificado é a condição do paciente, portanto, o uso da técnica é contraindicado em pacientes alérgicos, que tenham medo de animais, feridas abertas, problemas respiratórios, pacientes com baixa resistência, animais com zoonoses e pacientes agressivos que possam machucar os animais (PEREIRA et al., 2007).

Segundo Ferreira e Gomes (2017), tendo em vista os objetivos da TAA, esta terapia pode auxiliar o processo de humanização dos atendimentos, assim, partindo deste princípio, é necessário respeitar a decisão do paciente com a ideia de o animal fazer parte do tratamento ou não, trazendo em pauta o conforto e a motivação para adesão ao tratamento. Desta forma, ainda de acordo com os autores, há muitas técnicas que buscam promover a oferta de uma melhor condição física, mental e social do paciente, sendo que uma das técnicas é justamente a TAA como suporte à psicologia clínica.

A TAA é uma terapia mundialmente conhecida, uma vez que, esta técnica foi comprovada como eficaz no processo de socialização de pessoas, na psicoterapia, em 
tratamentos de pacientes com necessidades especiais e na diminuição da ansiedade de várias causas (PEREIRA et al., 2007).

O primeiro relato da aplicação da Terapia Assistida por Animais ocorreu em 1792, na Inglaterra, no retiro de York, onde William Tuke recorreu aos animais de fazenda para atuar como agentes facilitadores no contato com doentes mentais, partindo da ideia de que os animais contribuiriam para a realização das tarefas do dia a dia dos pacientes internados (ROVARIS E LEONEL, 2018). Tuke foi um dos primeiros a aderir à ideia da promoção de um tratamento mais humanizado, valorizando a reformulação do cuidar (ROVARIS E LEONEL, 2018).

No Brasil a TAA foi iniciada na década de 50 por Nise da Silveira, psiquiatra junguiana, brasileira; Nise iniciou um trabalho no Centro Psiquiátrico Pedro II, no Rio de Janeiro (AUTHAUSEN, 2006). Um dia, Nise se deparou com uma cachorrinha abandonada, pegou e levou-a próximo ao senhor Alfredo, um dos internos do hospital. Logo que se aproximou, Nise o questionou se gostaria de cuidar da cadelinha com muito cuidado, e a reposta de Alfredo foi positiva, então a cachorrinha foi nomeada como Caralâmpia (AUTHAUSEN, 2006).

Após este acontecimento, Nise percebeu que outros pacientes foram beneficiados com a presença dos animais; notou a contribuição dos animais desde a promoção de afeto até a noção da importância do cuidar (AUTHAUSEN, 2006).

O livro "Imagens do Inconsciente", publicado em 1982 por Nise da Silveira (1982, apud CAETANO, 2010, p. 2I), aborda a importância dos animais como coterapeutas:

"Excelentes catalisadores são os co-terapeutas não humanos. Desde a
adoção da pequena cadela Caralâmpia [...] verifiquei as vantagens da
presença de animais no hospital psiquiátrico. Sobretudo o cão reúne
qualidades que o fazem muito apto a tornar-se um ponto de referência
estável no mundo externo. Nunca provoca frustrações, dá incondicional
afeto sem nada pedir em troca, traz calor e alegria ao frio ambiente
hospitalar. Os gatos têm um modo de amar diferente. Discretos, esquivos,
talvez sejam muito afins com os esquizofrênicos na sua maneira peculiar
de querer bem."

As intervenções realizadas com animais são denominadas como: Atividade Assistida por Animais (AAA) e Terapia Assistida por Animais (TAA). De acordo 
com a Instituição Norte Americana Delta Society, a diferença das intervenções se dá de maneira que, a AAA busca proporcionar oportunidades para benefícios educacionais, motivacionais, de recreação e/ou terapêuticos, visando promover uma melhora na qualidade de vida, sendo realizada em uma variedade de ambientes por profissionais e/ou voluntários devidamente treinados, em conjunto com animais que obedecem a critérios especificados previamente (AUTHAUSEN, 2006).

Já a TAA, de acordo com a Instituição Delta Society, é exercida por profissionais de saúde/serviços humanos com experiência especializada e vinculada a prática profissional, uma vez que a TAA apresenta-se como uma forma de intervenção com objetivos estabelecidos. $\mathrm{O}$ animal obedece a critérios específicos, e pertence integralmente ao processo terapêutico, pois parte da concepção que esta intervenção tem o objetivo de melhorar o funcionamento emocional, social, físico e cognitivo (AUTHAUSEN, 2006).

Há diversas espécies de animais que podem ser utilizadas na TAA, como cães, gatos, cavalos, golfinhos e até tartarugas, entretanto, o cão permanece como o animal mais adotado à terapia, pois trata-se de uma espécie que demonstra maior índice de afetividade pelas pessoas (FERREIRA E GOMES, 2017).

Segundo Ferreira e Gomes (2017), outro ponto considerável é o fato do cão ser facilmente treinado, sendo capaz de estabelecer mecanismos positivos ao toque, o que facilita a aceitação por parte das pessoas. Ainda de acordo com os autores, entre as modalidades mais utilizadas por profissionais são a cinoterapia (terapia que utiliza cães) e a equoterapia (terapia que utiliza cavalos).

Como mencionado acima, a cinoterapia refere-se à terapia que utiliza o cão como integrante do processo terapêutico; quando utilizada com crianças, apresenta contribuições no processo de socialização, diminuição de ansiedade, diminuição do estresse e solidão, estimulando a troca de afeto (MEDEIROS E CARVALHO, 2008).

De acordo com Carvalho (2014), o cão auxilia o processo de estabelecer vínculo entre pacientes e terapeutas, além deste vínculo, há também o que é 
estabelecido entre o animal e o paciente, o que contribui para a manutenção de ações, como a gentileza, confiança, compaixão e identificação de uma amizade.

A cinoterapia vem despertando interesse maior dos profissionais $\mathrm{da}$ psicologia, pois possui validação científica e resultados satisfatórios, repercutindo assim, como forma de contribuição para pacientes com dificuldades em se expressar, lidar com traumas e com a timidez (FULBER, 20II).

A intervenção com o cão tem auxiliado no autocontrole, na autonomia e no autoconhecimento do paciente, desta forma, a cinoterapia tem apresentado resultados benéficos na aplicação com idosos, crianças e adolescentes, apresentando-se como um apoio a prática do psicólogo (CAETANO, 2010).

A equoterapia é uma prática antiga e foi reconhecida pelo Conselho Federal de Medicina em 1997, no qual, o cavalo contribui no tratamento de pessoas portadoras de necessidades especiais motoras e mentais (FERREIRA E GOMES, 2017).

Segundo Rovaris e Leonel (2018), por apresentar benefícios relacionados com a melhora biopsicossocial e melhora na afetividade, as principais indicações para o uso da técnica são para as pessoas com paralisia cerebral e transtorno do espectro autista. Entretanto, a equoterapia também vem sendo utilizada com pessoas com depressão, transtorno de ansiedade, dificuldades de aprendizagem e transtorno de déficit de atenção e hiperatividade (FERREIRA E GOMES, 2017).

\section{DESENVOLVIMENTO HUMANO: DO NASCIMENTO À ADOLESCÊNCIA}

O processo de transformação dos seres humanos inicia-se desde a concepção até o final da vida; ao longo desse processo há mudanças que são comuns entre os seres humanos, manifestando-se como mudanças padronizadas, assim, os bebês crescem e tornam-se crianças, que por sua vez crescem e tornam-se adultos, desta forma, os pesquisadores da área do desenvolvimento humano o caracterizam como um processo contínuo durante toda a vida (PAPALIA E FELDMAN, 2013). 
Segundo Papalia e Feldman (2013), os pesquisadores do desenvolvimento estudam as instâncias do eu, instâncias essas que são: físico, cognitivo e psicossocial. Ainda de acordo com os autores, dentro do desenvolvimento físico encontram-se etapas como o crescimento do cérebro, do corpo, o desenvolvimento das habilidades motoras e das capacidades sensoriais.

Já o desenvolvimento cognitivo é composto pelo desenvolvimento da aprendizagem, da linguagem, do pensamento, da criatividade, da memória, da atenção e do raciocínio (PAPALIA E FELDMAN, 2013). Ainda de acordo com Papalia e Feldman (2013), o desenvolvimento psicossocial aborda as emoções, a personalidade e a relação da pessoa com o meio, ou seja, abrange as relações sociais. Desta forma, os autores mencionam que, mesmo que haja uma separação entre o desenvolvimento físico, cognitivo e psicossocial, estas três áreas se conectam, portanto, cada área do desenvolvimento influencia a outra.

Há determinadas características que fazem parte dos estágios do desenvolvimento humano, posto isto, há estágios que fazem parte do desenvolvimento da criança e do adolescente (SOUZA et al., 2004). Desta forma, segundo os autores citados anteriormente, o primeiro estágio é o pré-natal, no qual, compreende o momento da concepção até o nascimento, neste estágio, decorre a constituição da estrutura e dos órgãos básicos do corpo, o crescimento físico é o mais acentuado neste período e há maior índice de vulnerabilidade com relação às influências ambientais. Segundo Souza et al. (2004), neste estágio, no que tange o desenvolvimento cognitivo, apresentam-se habilidades de aprender e lembrar, incluindo também a capacidade de responder aos estímulos sensoriais.

O segundo estágio do desenvolvimento é a primeira infância, que se inicia no nascimento até os 3 anos (XAVIER E NUNES, 2015). Ao nascer, os sentidos e sistemas do corpo da criança funcionam em diversos graus, com o aumento do cérebro há aceleração das habilidades motoras e do crescimento físico (XAVIER E NUNES, 2015). Ainda de acordo com os autores, neste estágio, com relação ao desenvolvimento cognitivo, nas primeiras semanas surgem capacidades de aprender 
e lembrar, a capacidade de resolução de problemas tende a surgir no final do segundo ano de vida, sendo que, o desenvolvimento da linguagem ocorre rapidamente.

De acordo com a abordagem piagetiana, do nascimento até os 2 anos de idade, a criança encontra-se no estágio sensório-motor, estágio este em que os bebês se realizam pela sua coordenação motora (GALLO E ALENCAR, 20I2).

A segunda infância abrange o período dos 3 aos 6 anos; com relação ao desenvolvimento físico, inicia-se a preferência pela utilização de uma das mãos, há o aprimoramento das habilidades motoras finas e gerais e há aumento da força física (SOUZA et al., 2004). Neste estágio, com relação ao desenvolvimento cognitivo, o pensamento da criança apresenta-se sob uma perspectiva egocêntrica, entretanto aumenta a consciência da opinião do outro, a memória e a linguagem neste estágio são aprimoradas e a inteligência se torna mais esperável (SOUZA et al., 2004).

Conforme a teoria de Piaget, a criança dos 2 aos 6 anos situa-se no estágio préoperatório, neste estágio a utilização do pensamento simbólico apresenta-se como principal característica, possibilitando assim a manifestação de novas maneiras de organização de opiniões (KRISCIELI E LIMA, 2002).

A terceira infância compreende a faixa etária dos 6 aos II anos; o desenvolvimento físico neste estágio apresenta como característica o fato da criança apresentar crescimento mais lento, apresenta aumento da força física, pois o desenvolvimento motor facilita a adesão a prática de mais atividades, podendo contribuir para o desenvolvimento de habilidades atléticas (XAVIER E NUNES, 2015). Ainda de acordo com os autores, na terceira infância, entre as características do desenvolvimento cognitivo inclui-se a diminuição do egocentrismo, a lógica começa a fazer parte do pensamento da criança, competências como linguagem e memória aumentam.

O estágio operatório concreto, de acordo com Piaget, engloba a idade dos 7 aos I2 anos aproximadamente, neste estágio, inicia-se a construção lógica, assim, neste momento a criança começa a perceber os diferentes pontos de vista, possibilitando a sua inserção em trabalhos em grupo, porém mesmo que neste estágio a criança 
adquira o pensamento lógico, ela ainda se baseia na realidade do aqui e o agora (MYERS, 2016).

Após a terceira infância, inicia-se a adolescência, que abarca a idade dos 12 anos até aproximadamente os 20 anos; o desenvolvimento físico neste período inclui características como o rápido crescimento físico, intercorrendo a maturidade reprodutiva, além de ocorrer outras mudanças rápidas e profundas (PAPALIA E FELDMAN, 2013). Segundo Papalia e Feldman (2013), no desenvolvimento cognitivo, características como o surgimento do raciocínio científico e a aptidão ao pensamento com termos abstratos são desenvolvidos, entretanto há persistência de comportamentos imaturos neste período.

De acordo com as ideias de Piaget, dos 12 a aproximadamente aos 19 anos de idade, o adolescente encontra-se no estágio operatório formal, neste estágio, o adolescente desenvolve o pensamento lógico além de demonstrar interesse pela utilização do pensamento abstrato, logo, a partir deste estágio, o adolescente consegue entender a temporalidade dos eventos que permeiam o seu dia a dia, por isso, ele não se limita mais ao aqui e o agora (FURNHAM, 2015).

\section{DESENVOLVIMENTO SOCIAL}

Desde o nascimento o bebê é caracterizado como uma criatura social, logo, em todos os estágios do desenvolvimento da criança novos hábitos de interação com o meio surgem, assim, independente da cultura, um dos vínculos mais intensos durante os primeiros estágios do desenvolvimento da criança é com os seus cuidadores (MYERS, 2016).

No que tange o desenvolvimento psicossocial, no período pré-natal o feto consegue reconhecer à voz da mãe e consequentemente dá prioridade à ela (PAPALIA E FELDMAN, 2013). Ainda de acordo com os autores, na primeira infância os vínculos afetivos são formados, tanto com os pais como com outras pessoas; neste estágio, a criança começa a ser mais autônoma e a disposição para a relação com outras crianças aumenta. 
Já na segunda infância, o entendimento das emoções e o autoconceito se tornam mais complexos, o desenvolvimento da identidade de gênero se torna presente $e$ as suas relações sociais são baseadas pela interação com a família, entretanto, neste momento a relação com outras crianças começa a se tornar mais importante (PAPALIA E FELDMAN, 2013).

$\mathrm{Na}$ terceira infância, o autoconceito apresenta-se com maior complexidade, impactando na autoestima (PIOVEZAN et al., 2018). Ainda de acordo com os autores, dado o estabelecimento de regras, há mudança gradativa na relação dos pais com a criança, neste estágio os colegas desempenham importância essencial.

Já na adolescência, torna-se presente a indagação da identidade, envolvendo a identidade sexual, neste estágio, as amizades podem influenciar de forma positiva ou negativa (PIOVEZAN et al., 2018). De acordo com Mayers (2016), na infância, o apego apresenta-se como a primeira realização social, sendo que, no final da infância, grande parte das crianças adquire o autoconceito, ou seja, a percepção de quem são elas.

$\mathrm{Na}$ teoria de Erik Erikson foram estabelecidas oito etapas que constituem o desenvolvimento psicossocial para exemplificar os acontecimentos no decorrer da vida (OLIVEIRA, 2006).

Desta forma, de acordo com a teoria de Erikson, o primeiro estágio é o do bebê (até I ano), caracterizado pelo surgimento de questões voltadas a confiança e a desconfiança (MYERS, 2016). Ainda de acordo com o autor, na teoria de Erikson, o segundo estágio é a infância (de I a 3 anos), neste estágio caraterísticas como a autonomia e a vergonha tornam-se presentes.

O terceiro estágio, na teoria de Erikson, é a pré-escola (de 3 a 6 anos), a iniciativa e a culpa emergem nesta etapa; já o quarto estágio abarca a idade dos 6 anos até a puberdade, nomeado como ensino fundamental, neste estágio questões vinculadas a competência e a inferioridade se tornam presentes (MYERS, 2016).

De acordo com as ideias de Erikson, o quinto estágio refere-se à adolescência (puberdade até os 20 anos), estágio este que diz respeito ao conflito entre a identidade e a confusão de papéis (OLIVEIRA, 2006). Neste estágio, a busca pela identidade 
torna-se presente, pois para o adolescente é necessário definir quem se é, considerando assim, aspectos do passado e do presente (PIOVEZAN et al., 2018).

A adolescência é marcada pela crise entre a identidade versus a confusão de papéis, neste estágio, o adolescente deixa de ser uma criança que depende do outro em todas as atividades, entretanto, ainda não se encontra na vida adulta $e$ independente, assim, o adolescente busca definir sua identidade, sendo necessário organizar suas necessidades, ideias e seus desejos (MARCH, 2010).

$\mathrm{Na}$ teoria de Henri Wallon, nomeada como teoria psicogenética, o autor diz que no desenvolvimento da criança, até determinado estágio, há predomínio do desenvolvimento biológico, entretanto, em determinado momento o desenvolvimento social alcança força maior, assim, para Wallon, o desenvolvimento social é fundamental, logo, o ser humano é organicamente social (FERREIRA E RÉGNIER, 20I0).

No primeiro ano da criança, ela se relaciona com o meio através da afetividade, logo, é a afetividade que faz com que o bebê interaja com as pessoas, este estágio é nomeado como impulsivo-emocional (GALVÃO, 1995). O segundo estágio (que vai até os 3 anos), de acordo com a teoria de Wallon, é sensório-motor e projetivo, que tem como característica o desenvolvimento da linguagem e da função simbólica (GALVÃO, 1995).

O terceiro estágio ainda de acordo com a teoria Walloniana é o personalismo (dos 3 aos 6 anos), neste estágio a principal característica é a formação da personalidade e da consciência, que se dá a partir das relações sociais (PAULA et al., 2020). Ainda de acordo com os autores, na teoria de Wallon, a partir dos 6 anos inicia-se o estágio categorial, este estágio é marcado pelo avanço intelectual, no qual o interesse pelo conhecimento é despertado na criança.

O último estágio na teoria de Wallon é a adolescência, neste estágio o adolescente vivencia a puberdade, a alteração na cognição e na noção da afetividade fazem-se presentes, tornando-se questionáveis assuntos que circundam as questões pessoais, existenciais e morais (GALVÃO, 1995). 
De acordo com a teoria sociocultural de Lev Semenovich Vygotsky, da mesma forma que Piaget, Vygotsky destaca a relação ativa da criança com o seu ambiente, assim, a teoria de Vygotsky baseia-se na ideia de que o desenvolvimento cognitivo da criança é orientado pelos processos culturais e sociais (PAPALIA E FELDMAN, 2013).

Para Vygotsky, o aprendizado da criança se dá através da interação social, portanto, ele enfatiza a importância da linguagem, pois acredita ser fundamental para a compreensão em relação ao mundo (PAPALIA E FELDMAN, 2013).

\section{HABILIDADES SOCIAIS}

O termo habilidades sociais é a titulação aos diferentes níveis de comportamentos sociais presentes no repertório das pessoas, assim essas habilidades sociais contribuem para a eficácia na interação das pessoas (DEL PRETTE E DEL PRETTE, 200I). As habilidades sociais também podem ser caracterizadas quando a pessoa expressa suas opiniões, atitudes, seus sentimentos, desejos, respeitando as suas ideias e as dos outros (CABALLO, 1996).

Há muitas definições sobre o que é um comportamento considerado socialmente habilidoso, logo, há dificuldade em aplicar uma definição exata sobre as habilidades sociais, pois para a definição, contextos como a cultura e os padrões de comunicação devem ser considerados, além de que a idade, o sexo, a educação e a classe social, também são questões a serem consideradas, assim, frente a uma situação, duas pessoas podem manifestar comportamentos totalmente diferentes (CABALlO, 1996).

De acordo com Del Prette e Del Prette (2009), as habilidades sociais são definidas como o agrupamento de comportamentos sociais do repertório do sujeito que facilita as relações interpessoais. Ainda de acordo com os autores, alguns comportamentos contribuem para o aperfeiçoamento social da pessoa, como a empatia, assertividade, comunicação, cooperação e o autocontrole.

Desta forma, a boa relação interpessoal na infância e adolescência, é essencial para o desenvolvimento destas, pois a partir das relações as habilidades sociais e a 
capacidade de enfrentamento mediante as situações conturbadas vão se estruturando (XAVIER E NUNES, 2015).

Para a compreensão das habilidades sociais, tais habilidades foram classificadas em categorias, assim, a primeira categoria é a de habilidades sociais de comunicação, esta categoria compreende elaborar e responder perguntas, iniciar, manter e finalizar conversas; já a segunda categoria é a de habilidades sociais de civilidade, que abarca noção de agradecer, de apresentar-se e despedir-se (DEL PRETTE E DEL PRETTE, 200I).

Segundo Del Prette e Del Prette (200I), a terceira categoria abrange as habilidades sociais de fazer e manter amizade, habilidades estas voltadas a iniciar e encerrar conversas, manter contatos e expressar sentimentos; a quarta categoria é a de habilidades sociais empáticas, esta habilidade compreende expressar apoio e refletir sentimentos. Ainda de acordo com os autores, a quinta categoria abrange as habilidades sociais assertivas, que refere-se à manifestação de opinião, aceitar e recusar pedidos, desculpar-se e admitir falhas, estabelecer e encerrar relacionamentos.

A sexta é a de habilidades sociais de autocontrole, que consiste em expressar emoções boas e ruins e controle de humor; já a sétima é a de habilidades sociais de solidariedade que abrange a oferta de ajuda, apoio e compartilhamento; a oitiva é a de habilidades sociais de manejar conflitos, que aborda a noção de exercer controle de emoções e identificar soluções e alternativas (DEL PRETTE E DEL PRETTE, 200I).

A nona habilidade, refere-se às habilidades sociais acadêmicas, que engloba o respeito às regras e o envolvimento nas atividades propostas; a décima compreende as habilidades sociais de expressar afeto, que refere-se à demonstração de afeto e de atenção ao outro; a décima primeira habilidade contém as habilidades sociais de coordenar grupo, que abrange a noção de organizar e explicar tarefas; a décima segunda habilidade refere-se as habilidades sociais de falar em público, que aborda questões como controle do tom de voz, olhar para o público e prestar suporte as possíveis dúvidas (DEL PRETTE E DEL PRETTE, 20or). 
Outro termo vinculado às habilidades sociais são as competências sociais, desta forma, competências sociais referem-se à capacidade que a pessoa possui para articular seus sentimentos, pensamentos e suas ações, logo, no processo de desenvolvimento das competências sociais na infância, para que a criança consiga manifestar as suas competências sociais, ela precisará de algumas habilidades sociais, como por exemplo, o autocontrole e a empatia (DEL PRETTE, 2005).

De acordo com Del Prette (2005), quando há ausência destas habilidades na infância, problemas emocionais e comportamentais podem se manifestar. Portanto, ainda de acordo com o autor, uma condição importante para o amadurecimento das habilidades sociais é a socialização, pois a partir da socialização a criança começa a entrar em contato com informações e ideias novas sobre o meio em que está inserida, assim, através desta interação, a aprendizagem das habilidades sociais tornam-se presentes.

A adolescência é um período do desenvolvimento no qual o adolescente deixa de ser criança, porém ainda não é adulto, logo essa transição para alguns adolescentes pode ser definida como um período no qual o estresse e os sentimentos ambíguos podem se manifestar (GUIZZO et al., 2018). Nesta fase do desenvolvimento novas tarefas são necessárias, exigindo assim, adaptações e habilidades sociais para enfrentar os desafios e as mudanças do processo do desenvolvimento (GUIZZO et al., 2018).

Dado o fato de que a aprendizagem das habilidades sociais se dá desde a infância, principalmente através da observação, um dos primeiros modelos de interação social que as crianças e os adolescentes acessam é a interação dos pais, sendo interações verbais (através da fala) e não verbais (através do contato visual) (SILVA, 202I).

De acordo com Silva (202I), é fundamental que a criança e o adolescente estejam inseridos em um ambiente que facilite o processo de aprendizagem das habilidades sociais, pois quando estes não estão inseridos em um contexto favorável, pode dificultar o desenvolvimento destas habilidades, podendo acarretar em déficit de fluência e desenvolvimento. 
Segundo Caballo (1996), o treinamento de habilidades sociais (THS) apresenta-se como uma das técnicas comportamentais mais utilizadas para o desenvolvimento de habilidades sociais. Portanto, ainda de acordo com o autor, o THS pode ser interpretado como uma medida de intervenção que busca ensinar habilidades e estratégias interpessoais, visando o desenvolvimento das habilidades sociais.

Desta forma, o treinamento de habilidades sociais na infância $e$ na adolescência inclui a utilização da modelagem, da instrução, do ensaio comportamental, do feedback e do reforço positivo (FRIEDBARG et al., 20II).

O THS, de acordo com a estruturação da técnica, se dá através de quatro elementos, sendo o primeiro referente ao treinamento de habilidades, o segundo refere-se à redução de ansiedade em situações sociais problemáticas, o terceiro aborda a reestruturação cognitiva e o quarto abrange o treinamento em solução de problemas (CABALLO, 1996).

\section{O BEM-ESTAR DOS ANIMAIS NA TAA}

Muitos estudos apontam os benefícios dos animais na vida de uma pessoa, especialmente quando este animal é o cachorro, pois ao acariciar o cão, pode ocorrer a diminuição do hormônio do estresse (cortisol), a redução da pressão arterial e a liberação da serotonina (SOUSA, 2016). Entretanto, além dos benefícios apresentados às pessoas, o animal também se beneficia com o contato com o ser humano, pois através da carícia, há aumento da endorfina, ocitocina e diminuição do cortisol e da pressão sanguínea (FISCHER et al., 2016).

A partir da presença dos cães na TAA, além da expectativa da experiência ser benéfica ao ser humano, é necessário atenção aos benefícios e prejuízos que esta interação pode acarretar no animal, logo, é necessário preocupar-se com o bem-estar da pessoa e do animal, porém, muitos estudos buscam mensurar os benefícios da TAA na qualidade de vidas das pessoas, esquecendo-se de olhar para o animal (VAZ, 2020). 
De acordo com Rocha (2015), muitas vezes o cão está inserido em contextos de interação terapêutica diariamente, assim, se não houver uma seleção rígida, acompanhamento contínuo do comportamento, consideração da história de vida e temperamento e o acompanhamento da qualidade de vida em suas residências, o animal pode estar vulnerável a estímulos estressores. Ainda de acordo com o autor, os animais utilizados na TAA devem ser considerados a partir de uma perspectiva holística, analisando as suas necessidades como um todo, considerando que o animal é muito mais que uma ferramenta terapêutica.

Entre os trabalhos que abordam o bem-estar animal, alguns autores consideram que o bem-estar tanto do animal quanto das pessoas devem ser equivalentes, ou seja, considera-se a valorização do bem-estar tanto da pessoa quanto do animal, entretanto, há autores que enfatizam o bem-estar da pessoa e não consideram o do animal (ROCHA, 2015).

Considera-se que o bem-estar do animal está vinculado ao bem-estar da pessoa, assim, há necessidade de que tanto a pessoa quanto o animal gozem da companhia um do outro, destacando a necessidade de considerar se o animal está confortável no local de atuação das atividades e que em algumas ocasiões o animal poderá não apresentar interesse (ROCHA, 2015).

\section{METODOLOGIA}

O presente trabalho refere-se a uma revisão bibliográfica, e teve como objetivo verificar, na literatura, como a TAA (Terapia Assistida por Animais) auxilia o desenvolvimento de habilidades sociais na infância e na adolescência.

De acordo com Gil (2002), a revisão bibliográfica é definida pela utilização de materiais já elaborados e publicados, como artigos científicos e livros, desta forma, a revisão bibliográfica se refere a um estudo baseado em pesquisas bibliográficas.

Para a elaboração deste trabalho foram utilizados livros e artigos publicados em revistas científicas, sendo estes materiais, consultados através das bases de dados SciELO (Scietific Eletronic Library Online), LILACS (Literatura Latino-Americana e do Caribe em Ciências da Saúde) e o CAPES. 
Como estratégia para a seleção dos materiais, foi realizado um levantamento em abril de 202I para quantificar os livros e artigos científicos sobre o tema. Na busca de materiais mediante o objetivo do trabalho, foram utilizados os operadores booleanos: "Terapia Assistida por Animais", “Terapia Assistida por Animais e Desenvolvimento Social”, “Terapia Assistida por Animais e Criança”, "Terapia Assistida por Animais e Adolescente" e "Terapia Assistida por Animais e Habilidades Sociais". Além disso, não foi fixado um período entre a publicação dos artigos.

Após a pesquisa pelos operadores booleanos mencionados acima, foram utilizados critérios de inclusão e exclusão. Entre os critérios de inclusão estão os artigos publicados na língua portuguesa, artigos brasileiros e revistas indexadas; entre os critérios de exclusão estão os artigos publicados em outra língua, publicados em outro país e artigos repetidos.

\section{PROCEDIMENTOS}

Para a busca dos materiais foi realizada a pesquisa pelo unitermo Terapia Assistida por Animais, e após esta tentativa, foram utilizados os seguintes operadores booleanos em conjunto: Terapia Assistida por Animais e Desenvolvimento Social, Terapia Assistida por Animais e Criança, Terapia Assistida por Animais e Adolescente e Terapia Assistida por Animais e Habilidades Sociais, sendo que na busca por esse último unitermo, não foram localizados artigos em nenhuma das bases de dados.

A primeira etapa de busca por artigos foi a pesquisa pelos operadores booleanos. $\mathrm{Na}$ base de dados SciELO foram localizados 22 artigos, no LILACS foram localizados 300 artigos e no CAPES foram localizados Io9 artigos.

A segunda etapa foi realizada os critérios de inclusão e exclusão e leitura dos títulos. Na base de dados SciELO foram selecionados io artigos, no LILACS foram selecionados 18 artigos e no CAPES foram localizados io artigos. 
A terceira etapa foi a leitura do resumo. $\mathrm{Na}$ base de dados SciELO foram selecionados 2 artigos, no LILACS foram selecionados 3 artigos e no CAPES foram localizados 2 artigos.

Desta forma, foram localizados 7 artigos que articulam com o objetivo do presente trabalho.

\section{Fluxograma}

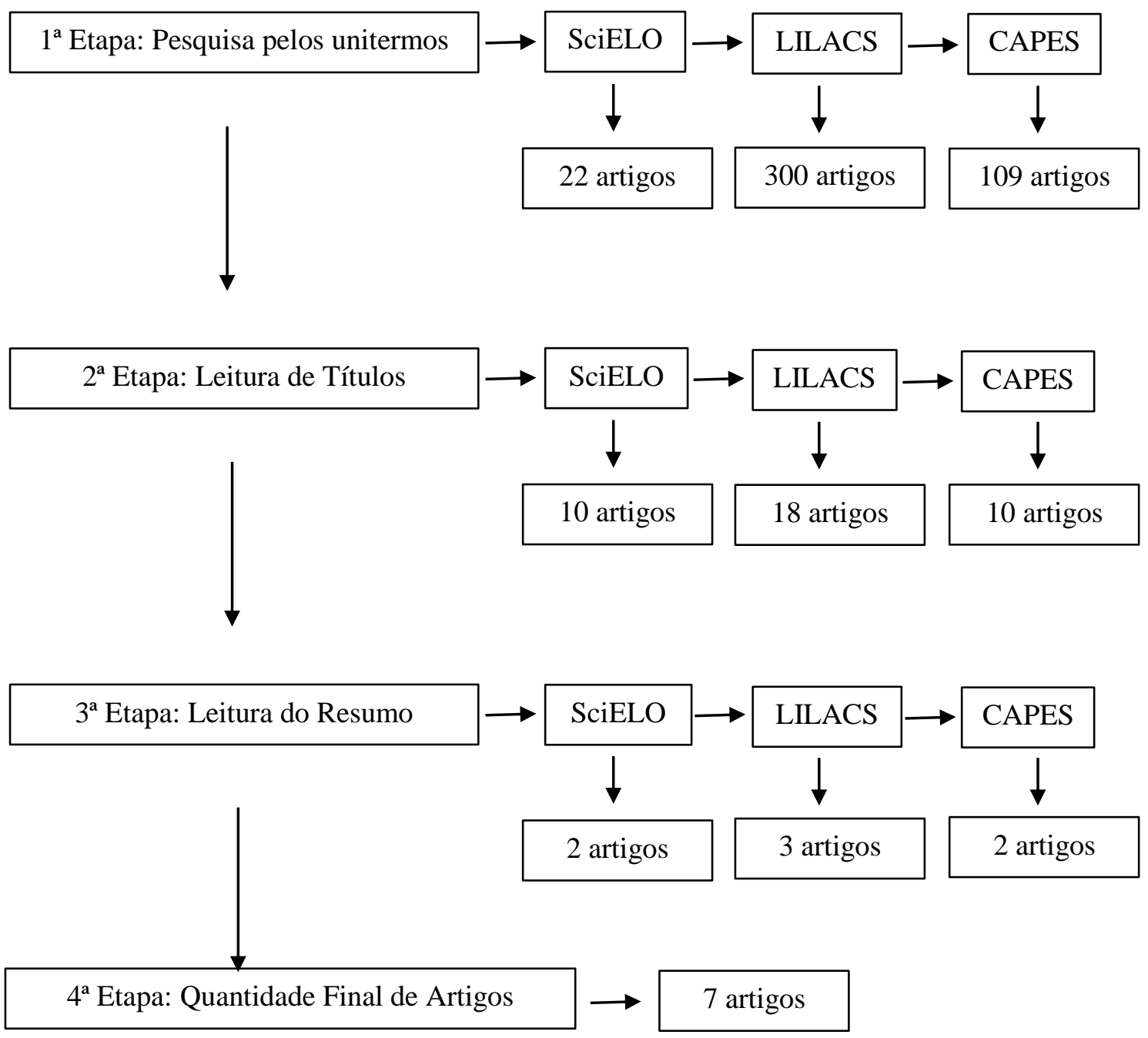

Fonte: As autoras (2021) 


\section{RESULTADOS}

\begin{tabular}{|c|c|c|c|}
\hline $\begin{array}{l}\text { Ano do } \\
\text { Artigo }\end{array}$ & Nome dos Autores & $\begin{array}{l}\text { Metodologia/ } \\
\text { Amostra }\end{array}$ & Resultado/ Conclusão \\
\hline 2017 & $\begin{array}{l}\text { MARINHO, J. R. } \\
\text { S.; ZAMO, R. S. }\end{array}$ & $\begin{array}{l}\text { Estudo } \\
\text { Qualitativo/ } \\
\text { Criança }\end{array}$ & $\begin{array}{l}\text { Neste estudo qualitativo foi apontada } \\
\text { a ideia de a TAA ser uma } \\
\text { intervenção potencial para ser } \\
\text { utilizada com crianças com } \\
\text { dificuldades sociais, cognitivas e } \\
\text { físicas. Porém o artigo menciona a } \\
\text { necessidade de mais estudos sobre o } \\
\text { tema. }\end{array}$ \\
\hline 2016 & $\begin{array}{l}\text { MOREIRA, R. L. } \\
\text { et al. }\end{array}$ & $\begin{array}{l}\text { Estudo } \\
\text { Qualitativo/ } \\
\text { Criança e } \\
\text { Adolescente }\end{array}$ & $\begin{array}{l}\text { Neste estudo qualitativo foi } \\
\text { percebido que a prática da TAA é } \\
\text { reconhecida como benéfica, mas os } \\
\text { pacientes possuem dificuldades em } \\
\text { compreender o real objetivo } \\
\text { terapêutico, pois associam como } \\
\text { diversão e distração. Entretanto, o } \\
\text { artigo demonstra que a TAA pode se } \\
\text { tornar uma modalidade efetiva na } \\
\text { promoção de saúde de crianças e } \\
\text { adolescentes com câncer, pois foi } \\
\text { constatado que a TAA proporcionou } \\
\text { a redução de ansiedade e do trauma } \\
\text { da hospitalização nos pacientes, } \\
\text { facilitando o processo de socialização } \\
\text { das crianças com outras crianças e } \\
\text { com os profissionais de saúde. }\end{array}$ \\
\hline 2014 & $\begin{array}{l}\text { PORTO, J. R.; } \\
\text { QUATRIN, L. B. }\end{array}$ & $\begin{array}{l}\text { Estudo de Caso/ } \\
\text { Adolescente }\end{array}$ & $\begin{array}{l}\text { Neste estudo de caso foi abordado } \\
\text { que a TAA apresenta-se como uma }\end{array}$ \\
\hline
\end{tabular}




\begin{tabular}{|c|c|c|c|}
\hline & & & $\begin{array}{l}\text { intervenção terapêutica capaz de } \\
\text { facilitar a melhora no desempenho } \\
\text { motor e melhora no aspecto } \\
\text { socioafetivo do adolescente com } \\
\text { paralisia cerebral. }\end{array}$ \\
\hline 2013 & $\begin{array}{l}\text { MARILENO, G. S.; } \\
\text { JARDIM, D. P. }\end{array}$ & $\begin{array}{l}\text { Pesquisa } \\
\text { Descritiva/ } \\
\text { Criança }\end{array}$ & $\begin{array}{l}\text { Nesta pesquisa descritiva, a TAA foi } \\
\text { abordada como uma técnica de } \\
\text { humanização no atendimento à } \\
\text { criança hospitalizada, ressaltando } \\
\text { que o relacionamento entre as } \\
\text { crianças e os animais favorecem } \\
\text { nelas a habilidade de se comunicar } \\
\text { com outras pessoas, desenvolvendo a } \\
\text { interpretação da linguagem dos } \\
\text { gestos. Os autores mencionam a } \\
\text { necessidade de novas pesquisas sobre } \\
\text { a técnica. }\end{array}$ \\
\hline 2011 & ZAGO, L. G. et. al. & $\begin{array}{l}\text { Estudo de Caso/ } \\
\text { Criança }\end{array}$ & $\begin{array}{l}\text { Neste estudo de caso foi apresentado } \\
\text { uma melhora funcional com relação } \\
\text { ao autocuidado, mobilidade e função } \\
\text { social, ressaltando a TAA como um } \\
\text { recurso que proporciona melhor } \\
\text { desempenho das atividades } \\
\text { funcionais da criança, devido ser uma } \\
\text { intervenção que apresenta-se como } \\
\text { lúdica e estimulante. O autor destaca } \\
\text { o fato de a TAA ser uma intervenção } \\
\text { terapêutica pouco pesquisada. }\end{array}$ \\
\hline 2009 & $\begin{array}{l}\text { KOBAYASHI, C. } \\
\text { S. et al. }\end{array}$ & $\begin{array}{l}\text { Relato de Caso/ } \\
\text { Criança }\end{array}$ & $\begin{array}{l}\text { Neste relato de caso foi constatado } \\
\text { resultados positivos da intervenção }\end{array}$ \\
\hline
\end{tabular}




\begin{tabular}{|c|c|c|c|}
\hline & & & $\begin{array}{l}\text { da TAA através do projeto "Amicão" } \\
\text { entre os pacientes, acompanhantes e } \\
\text { profissionais de saúde, ressaltando a } \\
\text { felicidade dos pacientes e } \\
\text { profissionais de saúde ao encontrar } \\
\text { com o cão, repercutindo também no } \\
\text { interrompimento do choro das } \\
\text { crianças e na formação de grupos } \\
\text { para ver o cão, que só se desfaz } \\
\text { quando ele vai embora. }\end{array}$ \\
\hline 2005 & $\begin{array}{l}\text { BUSSOTTI, E. A. } \\
\text { et. al. }\end{array}$ & $\begin{array}{l}\text { Estudo de Caso/ } \\
\text { Adolescente }\end{array}$ & 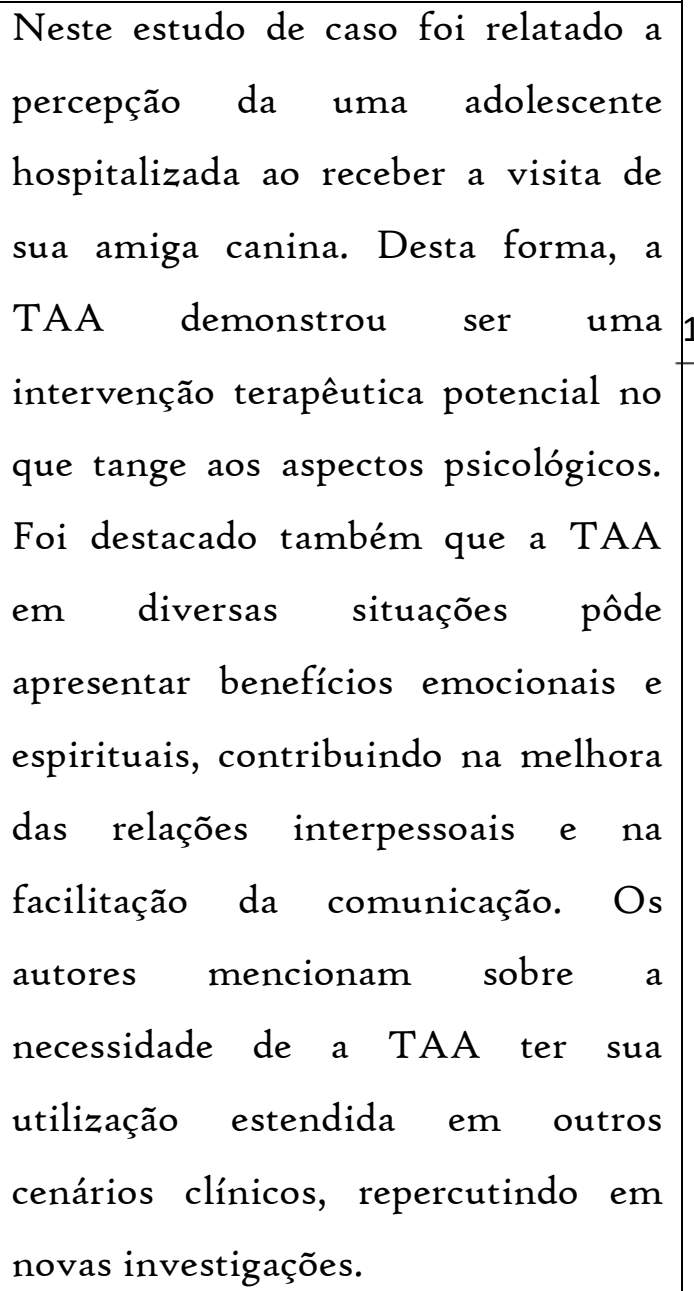 \\
\hline
\end{tabular}

Fonte: As autoras (2021) 


\section{DISCUSSÃO}

O presente trabalho identificou como um dos resultados o artigo de Marinho e Zamo (2017), cujo objetivo foi identificar os benefícios que a TAA pode gerar no tratamento com crianças com comprometimento neurodesenvolvimental, teve como resultado a ideia de a TAA ser uma intervenção potencial para ser utilizada com crianças com dificuldades sociais, cognitivas e físicas. Corroborando com o presente resultado, Rose, Cannas e Cantielo (2011), afirmam que a TAA apresenta cinco mecanismo de ação: o primeiro mecanismo é o afetivo-relacional, que reforça a importância do vínculo humano-animal, reforçando que, o vínculo emocional interfere diretamente em melhores resultados; o segundo mecanismo é o estímulo psicológico, no qual o vínculo estabelecido entre o humano-animal age na psique humana como um estímulo, facilitando a melhora no comportamento social e nos aspectos cognitivos; o terceiro mecanismo é o recreativo, na qual as brincadeiras estabelecidas com o animal favorecem a autoestima, atuando na diminuição do isolamento, proporcionando mudanças positivas no humor; o quarto mecanismo é o psicossomático, no qual postula que, a partir dos mecanismos anteriores, as intervenções com animais têm expressivas implicações psicossomáticas; o quinto mecanismo é o físico, no qual refere que o físico pode ser utilizado de diversas formas mediante as necessidades do paciente.

A presente pesquisa evidenciou o estudo de Moreira et al. (2016) que objetivou compreender a impressão dos profissionais da equipe de enfermagem e dos responsáveis por crianças e adolescentes com câncer sobre a Terapia Assistida por Animais. Obteve-se como resultado que a prática da TAA é benéfica, mas os pacientes possuem dificuldades em compreender o real objetivo terapêutico, pois associam como diversão e distração. Entretanto, a TAA pode se tornar uma modalidade efetiva na promoção de saúde de crianças e adolescentes com câncer, pois foi constatado que a TAA proporcionou a redução de ansiedade e do trauma da hospitalização nos pacientes. Para ratificar o presente resultado, o trabalho de Belletato e Banhato (2019), identificou que o contato com os animais pode facilitar a redução da pressão arterial e a redução do hormônio do estresse, o cortisol, podendo 
aumentar a quantidade de ocitocina, proporcionando também a sensação de segurança para a pessoa.

A presente pesquisa também verificou que no estudo de Porto e Quatrin (2014), cujo objetivo foi analisar a consequência da TAA sobre as condições motoras e socioafetivas de um adolescente com paralisia cerebral, que a TAA se apresenta como uma intervenção terapêutica que facilita a melhora no desempenho motor e melhora no aspecto socioafetivo do adolescente. Corroborando com o presente achado, Dotti (2005) afirma que entre os benefícios da TAA utilizada com adolescentes encontram-se a motivação, sentimento de segurança, diversão, troca de afeto e a redução da ansiedade e da solidão, ressaltando que o convívio com o cão facilita a atenção e a concentração do paciente aos exercícios de desenvolvimento da parte motora, contribuindo no desempenho das atividades.

No trabalho de Marinelo e Jardim (2013), no qual o objetivo foi realizar uma pesquisa sobre estratégias de humanização manuseadas pela equipe de saúde com o paciente pediátrico no ambiente hospitalar, verificou-se que a TAA apresenta-se como uma técnica de humanização no atendimento à criança hospitalizada, ressaltando que o relacionamento entre as crianças e os animais favorecem nelas a habilidade de se comunicar com outras pessoas, desenvolvendo a interpretação da linguagem dos gestos. A comunicação se faz necessária no tratamento do paciente, porém iniciar uma conversa nem sempre é uma tarefa simples, desta forma, a TAA se apresenta como uma medida válida no estabelecimento da comunicação entre o paciente e o profissional (SERBIN, 200I). Ainda de acordo com o autor, é comum ver pacientes com dificuldades em falar e se expressar, mas ao entrarem em contato com o animal, eles podem acariciar, alimentar os animais e brincar, sentindo-se incentivados a emitir expressões vocais, confirmando, portanto, com o resultado da presente pesquisa.

É importante salientar que o vínculo estabelecido entre as crianças e o cachorro, contribui para a autoestima, autonomia, noção de responsabilidade e para o estabelecimento da confiança, auxiliando na diminuição do estresse e no desenvolvimento contínuo da criança (HAVENER et al., 20oI). 
No trabalho de Zago et al. (2011) identificado como resultado da presente pesquisa, no qual o objetivo foi verificar a influência da TAA na funcionalidade de uma criança com Diplegia Espástica (um tipo de paralisia cerebral), verificou-se como resultado uma melhora funcional com relação ao autocuidado, mobilidade e função social, ressaltando a TAA como uma intervenção que se apresenta como lúdica e estimulante. De acordo com San Joaquín (2002), os benefícios da TAA são variados, como estímulo à memória, sensação de segurança, motivação, recuperação da autoestima, estímulo à prática de exercícios e a socialização, confirmando, portanto, com o resultado da presente pesquisa.

Verificou-se que no estudo de Kobayashi et al. (2009), cujo objetivo foi propiciar aos pacientes internados no Hospital São Paulo uma experiência positiva ao receberem um cachorro para sessões de TAA através do projeto “Amicão”, foram constatados resultados positivos da utilização da TAA entre os pacientes, acompanhantes e profissionais de saúde, ressaltando a felicidade dos pacientes e profissionais de saúde ao encontrar com o cão, o que também repercutiu no interrompimento do choro das crianças e na formação de grupos para ver o cão. Ratificando com o estudo, pode-se considerar o animal um facilitador no desenvolvimento de habilidades sociais, pois facilita a comunicação entre as pessoas, atuando na diminuição da ansiedade e do sentimento de solidão (BELLETATO E BANHATO, 2019), assim, por apresentar contato físico seguro, as relações interpessoais se estabelecem com segurança (LIMA E SOUSA, 2004).

No estudo de Bussotti et al. (2005), no qual o objetivo foi acessar a percepção de uma adolescente com leucemia e da sua mãe a respeito da visita do seu cão durante a hospitalização, demonstrou-se que a TAA é uma intervenção terapêutica potencial no que tange aos aspectos psicológicos, pois a TAA em diversas situações pôde apresentar benefícios emocionais e espirituais, contribuindo na melhora das relações interpessoais e na facilitação da comunicação. Desta forma, de acordo com a categorização das habilidades sociais de Del Prette \& Del Prette (200I), uma das habilidades sociais é a habilidade social de comunicação, categoria que baseia-se na 
elaboração e respostas de perguntas e na habilidade de iniciar, manter e encerrar conversas.

De acordo com os resultados apresentados, a TAA apresenta-se como uma intervenção terapêutica potencial no que tange os aspectos sociais, cognitivos e físicos, contribuindo para a habilidade de comunicação e melhora do desenvolvimento das relações interpessoais.

Os resultados apontaram a necessidade de mais estudos sobre o tema, ressaltando a ideia de que estudos na área podem facilitar o contato de psicólogos com a técnica ou de indicarem este modelo de tratamento.

\section{CONCLUSÃO}

Este trabalho abordou a relação da Terapia Assistida por Animais (TAA) no desenvolvimento das habilidades sociais na infância e adolescência, ou seja, buscou identificar a contribuição dos animais no desenvolvimento de habilidades sociais da criança e do adolescente.

Com base nos resultados apresentados e discutidos, a TAA apresenta-se como uma intervenção que facilita a socialização; os resultados também indicaram que a TAA atua diretamente no bom desempenho das relações interpessoais, pois facilita a comunicação entre as pessoas, facilitando a troca de afeto, noção de segurança, a atenção e a redução da ansiedade.

Por meio deste trabalho foi possível identificar a contribuição da TAA no desenvolvimento das habilidades sociais na infância e na adolescência, entretanto, é perceptível a necessidade de mais pesquisas sobre o tema mediante o fato de terem poucas teorias que associem ambas as ideias.

Por ora, foi possível perceber que a TAA se apresenta como uma intervenção de tratamento potencial, entretanto, para que sejam mensurados outros resultados, se faz necessário mais estudos sobre o tema para que assim contribuam com a divulgação desta modalidade, facilitando a adesão desta forma de intervenção por mais psicólogos. 


\section{REFERÊNCIAS}

ALTHAUSEN, S. Adolescentes com síndrome de Down e cães: compreensão e possibilidades de intervenção. Dissertação (Mestrado), São Paulo, 2006.

BELLETATO, L.; BANHATO, E. F. C. Transtorno de ansiedade social (TAS) ou fobia social: contribuições da terapia assistida por animais (TAA). Cadernos de Psicologia - CESJF - v.I n.I p.96-II4, Juiz de Fora, 20I9.

BUSSOTI, E. A.; LEÃO, E. R.; CHIMENTÃO, D. M. N.; SIlVA, C. P. R. Assistência individualizada: "Posso trazer meu cachorro?". Rev Esc Enferm USP; 39(2):195-201; 2005.

CABALLO, V. E. Manual de técnicas de terapia e modificação do comportamento. São Paulo: Santos Livraria Editora, 1996.

CAETANO, E. C. S. As contribuições da TAA: terapia assistida por animais à psicologia. Monografia (Trabalho de conclusão de curso) - Universidade do Extremo Sul Catarinense, Criciúma, 2010.

CAPOTE, P. S. O.; COSTA, M. P. R. Terapia Assistida por Animais (TAA): aplicação no desenvolvimento psicomotor da criança com deficiência intelectual. São Carlos, SP: Editora UFSCar, 2oir.

CARVALHO, I. A. Cinoterapia como recurso terapêutico para crianças com Transtorno do Espectro Autista: Uma revisão assistemática da literatura. Monografia do curso de psicologia. Universidade Federal do Rio Grande do Sul Instituto de Psicologia Porto Alegre, 20I4.

DEL PRETTE, A.; DEL PRETTE, Z. A. P. Psicologia das relações interpessoais: Vivências para o trabalho em grupo. Petrópolis, RJ: Vozes, 20oI.

DEL PRETTE, Z. A. P. Psicologia das habilidades sociais na infância: teoria e prática. Petrópolis, RJ: Vozes, 2005.

DEL PRETTE, Z. A. P; DEL PRETTE, A. Psicologia das habilidades sociais: Terapia e educação. Petrópolis, RJ: Vozes, 2009.

DOTTI, J. Terapia e Animais: Atividade e Terapia Assistida por Animais - A/ TAA: práticas para organizações, profissionais e voluntários. São Paulo: PC, 2005.

FERREIRA, A. P. S.; GOMES, J. B. Levantamento histórico da terapia assistida por animais. Revista Multidisciplinar Pey Këyo Científico - ISSN 2525-8508, Vol. 3, No I. 2017. 
FERREIRA, A. L.; RÉGNIER, N. M. A. Contribuições de Henri Wallon à relação cognição e afetividade na educação. Educar, Curitiba, Editora UFPR, n. 36, p. 21-38, 2010.

FISCHER, M. L.; ZANATTA. A. A.; ADAMI, E. R. Um olhar da bioética para a zooterapia. Revista Latinoamericana de Bioética, vol. I6, núm. I, janeiro- junho, pp.174-197, 2016.

FONSACA, K.; ARAÚJO, D. S. L. Desenvolvimento cognitivo: um estudo de caso na segunda infância. X Congresso Nacional de Educação - EDUCARE, Pontifícia Universidade Católica do Paraná, Curitiba, 201.

FRIEDBERG, R. D.; MCCLURE, J. M.; GARCIA, J. H. Técnicas de terapia cognitiva para crianças e adolescentes. Porto Alegre: Artmed, 2011.

FULBER, S. Atividade e Terapia Assistida por Animais. Trabalho de conclusão em medicina. Faculdade veterinária. Universidade Federal do Rio Grande do Sul, 201 .

FURNHAM, A. 50 Ideias de psicologia que você precisa conhecer. I. ed. - São Paulo: Planeta, 2015.

GAllO, A. E.; AlENCAR, J. S. A. Psicologia do Desenvolvimento da Criança. CESUMAR - Centro Universitário de Maringá - Núcleo de Educação à distância, Maringá - PR, 2012.

GALVÃO, I. Henri Wallon: uma concepção dialética do desenvolvimento infantil. 4. ed. Petrópolis, RJ; Vozes, 1995.

GIL, A. C. Como elaborar projetos de pesquisa. 4 ed. Editora Atlas. São Paulo, 2002.

GUIZZO, C. S. P.; PRETTE, A. D.; PRETTE, Z. A. P.; LEME, V. B. R. Programa de habilidades sociais para adolescentes em preparação para o trabalho. Psicologia Escolar e Educacional, SP. Volume 22, Número 3, Setembro/Dezembro de 2018.

HAVENER, L.; GENTES, L.; THALER, B.; MEGEL, M. E.; BAUN, M. M.; DRISCOLL, F. A.; BEIRAGHI, S.; AGRAWAL, S. The effects of a companion animal on distress in children undergoing dental procedures. Issues Compr. Pediatr. Nurs, Omaha, v. 24, n. 2, p. 137-152, 2001.

KOBAYASHI, C. T.; USHIYAMA, S. T.; FAKIH F. T.; ROBLES, R. A. M.; CARNEIRO, I. A.; CARMAGNANI, M. I. S. Desenvolvimento e implantação de Terapia Assistida por Animais em hospital universitário. Rev Bras Enferm, jul-ago; 62(4): 632-6; Brasília 2009. 
LIMA, M.; SOUSA, L. A Influência Positiva dos Animais de Ajuda Social. Interacções, 156 - 174; 2004.

MARCH, R. A. Formação da identidade e institucionalização: Uma relação possível? Monografia do curso pós-graduação de psicologia. Universidade Cândido Mendes, Rio de Janeiro, 2010.

MARINELO, G. S.; JARDIM, D. P. Estratégias lúdicas na assistência ao paciente pediátrico: aplicabilidade ao ambiente cirúrgico. Rev. SOBECO, São Paulo. abr./jun. 18(2): 57-66; 2013.

MARINHO, J. R. S.; ZAMO, R. S. Terapia assistida por animais e transtornos do neurodesenvolvimento. Estudos e Pesquisa em Psicologia. Rio de Janeiro, 2017.

MEDEIROS, A. J. S.; CARVALHO, S. D. Terapia assistida por animais a crianças hospitalizadas: revisão bibliográfica. Campinas, SP: Departamento de enfermagem, Faculdade de Ciências Médicas, Universidade Estadual de Campinas, 2008.

MOREIRA, R. L.; GUBERT, F. A.; SABINO, L. M. M.; BENEVIDES, J. L.; TOME, M. A. B. G.; MARTINS, M. C.; BRITO, M. A. Terapia assistida com cães em pediatria oncológica: percepção de pais e enfermeiros. Revista Brasileira de Enfermagem, 2016.

MYERS, D. G. Psicologia. 9. ed. Rio de Janeiro: LTC, 2016.

OLIVEIRA, M. C. S. L. Identidade, narrativa e desenvolvimento na adolescência: uma revisão crítica. Psicol. estud. vol.ıI no.2 Maringá May/Aug. 2006.

PAPALIA, D. E.; FELDMAN, R. D. Desenvolvimento Humano. 12. ed. - Porto Alegre: AMGH, 2013.

PAUlA, M. C.; GUIMARÃES, G. T. D.; NASCIMENTO, M. M. S.; VIALI, L. Contribuições de Henry Wallon: o papel da emoção na aprendizagem. RBSE: Revista Brasileira de Sociologia da Emoção, v. I9, n. 56, p. I8I-I92, agosto de 2020.

PEREIRA, M. J. F.; PEREIRA, L.; FERREIRA, M. L. Os Benefícios da Terapia Assistida dos Animais: uma revisão bibliográfica. Saúde Coletiva, vol. 4, núm. I4, pp. 62-66 Editorial Bolina, São Paulo, Brasil, abril-maio, 2007.

PIOVEZAN, J.; OTTONELli, J. C.; BORDIN, J. B.; PIOVESAN, L. Psicologia do desenvolvimento e da aprendizagem. I. ed. Universidade Federal de Santa Maria - RS, 2018. 
PORTO, J. R.; QUATRIN, L. B. Efeito da Terapia Assistida por Animais nos aspectos motores e interação socioafetiva de um adolescente com paralisia cerebral: um estudo de caso. ConScientiae Saúde, 13(4):625-632, São Paulo, Brasil, 2014.

ROCHA, C. F. P. G. Avaliação comportamental e endócrina do nível de estresse de cães participantes de intervenções assistida por animais. Dissertação de Programa Pós-Graduação em Psicologia Experimental para obtenção do título em Mestre em Psicologia. Universidade de São Paulo. São Paulo, 20ı5.

ROSE, P.; CANNAS, E.; CANTIELLO, P. R. Donkey-assisted rehabilitation program for children: a pilot study. Ann Ist Super Sanità; Vol. 47, No. 4: 39I-396; 2011.

ROVARIS, J. D. D. L.; LEONEL, W. H. D. S. Terapia Assistida por animais no auxílio ao processo educacional de crianças com deficiência intelectual. Revista CESUMAR, v. 23, n. 2, p. 341-357, jul./dez. 2018.

SAN JOAQUÍN, M. P. Z. Terapia asistida por animales de compañía. Bienestar para el ser humano, 2002.

SERBIN, S. The Bond: Newsletter of the SF/SPCA Animal Assisted Therapy Program. San Francisco, v. 3, n. I, p.I, feb. 2001.

SILVA, L. T. B. Avaliação funcional e de habilidades sociais de adolescentes com paralisia cerebral. Universidade Federal de São Carlos. Centro de educação e ciências humanas. Programa de pós-graduação em educação especial. São Carlos, 2021.

SOUSA, N. K. L. Terapia facilitada por cães: Estudo de caso. Tese de trabalho de conclusão de curso no Centro de Ciências Agrárias da Universidade Federal da Paraíba, para obtenção do título de Bacharel em Zootecnia. Areia-PB, 2016.

SOUZA, J. C.; FRAGA, L. L.; OLIVEIRA, M. R. O.; BUCHARA, M. S.; StRAliOTtO, N. C.; ROSÁRIO, S. P.; REZENDE, T. M. Atuação do Psicólogo Frente aos Transtornos Globais do Desenvolvimento Infantil. Psicologia ciência e profissão, 24 (2), 24-31, 2004.

VAZ, J. N. R. Os possíveis estresses causados em animais utilizados em terapias: cinoterapia e equoterapia. Revista Científica Doctum: Multidisciplinar. DOCTUM. Caratinga. v. I, n. 4. ISSN: 2595-1629, 2020.

XAVIER, A. S.; NUNES, A. I. B. L. Psicologia do Desenvolvimento. 4. ed. Revisada e ampliada, Fortaleza - Ceará, 2015. 
ZAGO, L. G.; FINGER, A. V.; KINTSCHNER, F. M. A influência da terapia assistida por animais na funcionalidade de uma criança com diplegia espástica: um estudo de caso. ConScientiae Saúde, ro(3):563-571, São Paulo, Brasil, 2orı. 